Cultures \& Conflits

08 | hiver 1992

Les conflits après la bipolarité

\title{
Le Khmer rouge : Homo bellicus versus homo economicus
}

Christian Leverchy

\section{(2) OpenEdition \\ 12 Journals}

Édition électronique

URL : http://journals.openedition.org/conflits/525

DOI : $10.4000 /$ conflits.525

ISSN : $1777-5345$

Éditeur :

CCLS - Centre d'études sur les conflits lilberté et sécurité, L'Harmattan

Édition imprimée

Date de publication : 6 décembre 1992

ISSN : 1157-996X

Référence électronique

Christian Leverchy, "Le Khmer rouge : Homo bellicus versus homo economicus », Cultures \& Conflits

[En ligne], 08 | hiver 1992, mis en ligne le 27 janvier 2003, consulté le 30 mars 2021. URL : http://

journals.openedition.org/conflits/525; DOI : https://doi.org/10.4000/conflits.525

Ce document a été généré automatiquement le 30 mars 2021.

Creative Commons License 


\title{
Le Khmer rouge : Homo bellicus versus homo economicus
}

\author{
Christian Leverchy
}

1 Conduit par un chef parlant en termes apocalyptiques de construction d'une société nouvelle, ou plutôt de destruction de l'ordre ancien; doté d'un régime fondé sur une idéologie parfaitement abjecte prônant la haine raciale, force est de rappeler que Pol Pot et les Khmers rouges de l'Angkar ont perpétré, de 1975 à 1978, des actes dont la nature et l'ampleur défient l'imagination ${ }^{1}$. Il est donc bien difficile intellectuellement de traiter l'époque du Kampuchéa démocratique comme n'importe quelle autre période du passé, comme une "page d'histoire" parmi d'autres. Face à cette réalité tout comme à l'horreur nazi, les capacités de l'historien semblent bien dérisoires². Dès lors, comment peut-on aborder avec justesse, voire avec "objectivité" cette faction cambodgienne, son attitude politique dans le processus de paix des accords de Paris, sa légitimité dans la société khmère contemporaine et plus largement son avenir politique? En dénonçant les horreurs passées, on voudrait croire que les compterendus des analystes (politologues, historiens, journalistes, responsables d'ONG) servent de témoignage pour le présent et pourquoi pas d'avertissement pour l'avenir. Néanmoins, il apparait que ce mouvement politique continue d'exister, de bénéficier d'une certaine aura à travers tout le pays et de disposer d'une réelle capacité de nuisance. Néanmoins, le regain de dynamisme guerrier des Khmers rouges, à la fin de l'année 1992, ne doit pas nous laisser croire à une marche triomphale des hommes de Pol Pot sur Phnom Penh. Ils demeurent, tout au moins pour le moment, incapables de reprendre le pouvoir. De plus, l'extension territoriale n'est pas, loin s'en faut, leur objectif primordial.

Le Khmer rouge : Homo bellicus

2 Même s'il conviendrait de réexaminer l'ampleur des massacres et leur répartition territoriale ${ }^{3}$, l'horreur passée ne doit pas nous laisser croire un seul instant que nous avons face à nous, uniquement, un léviathan sanguinaire. Ce serait une faute politique gravissime de sous-estimer les Khmers rouges, le degré de sophistication de leur organisation politique, la cohérence de leurs discours de propagande, leurs capacités à 
s'adapter à de nouveaux contextes, en bref leur intelligence à faire la guerre. Si chacun ne veut retenir de leurs tactiques que l'emploi de la violence (offensives militaires sur Kompong Thom, exécutions de civils vietnamiens et d'officiels de l'Etat du Cambodge (EdC), menaces sur les personnels de l'Autorité provisoire des Nations-unies au Cambodge (APRONUC), pillage des villages), en bref leurs capacités à être des Homo Bellicus, nous prenons le risque de nous contenter de ne prendre en compte que les formes symboliques de leur propédeutique de la terreur. Certes, comme en témoigne des documents récemment saisis, les Khmers rouges ont toujours pour perspective de reconquérir le pouvoir à Phnom Penh, voire de placer à la tête du nouvel Etat le Prince Norodom Sihanouk ${ }^{4}$ mais c'est également une guérilla affaiblie. En dépit de plus de 1 500 violations du cessez-le-feu, la carte des conflits n'a guère évolué et les combats se limitent à la périphérie des bastions sans que chacun puisse agrandir ses terroirs.

La mise en place des unités de l'APRONUC nous a, en effet, permis d'apprendre que la partie du Kampuchéa démocratique (PKD) craignait qu'une ouverture au monde lui soit fatale, de mesurer son manque de cadres compétents, aggravé par la centralisation de système de décision, et de réévaluer à la baisse le nombre ( 8 à 12000$)$ de ses combattants voire leur efficacité au feu ${ }^{5}$. Il n'en reste pas moins très difficile de savoir si les Khmers rouges se battent tout simplement pour leur survie où si leur stratégie présente, refus de la mise en oeuvre de la Phase II du cessez-le-feu (désarmement, cantonnement des unités), vise d'abord à gagner du temps, en comptant sur l'usure des Nations-unies pour engranger de nouveaux gains politiques. Même s'ils ont le sentiment que le processus de l'ONU revêt l'aspect d'une coalition anti-khmère rouge, les hommes de Pol Pot ont su disséquer le plan de paix, signé à Paris le 23 octobre 1991, et le tourner à leur profit. Il est vrai qu'à défaut de bénéficier de nouveaux armements chinois, les Khmers rouges peuvent compter sur la logistique diplomatique de Beijing. Mais plus généralement, ils peuvent profiter des lacunes que montre chaque jour un peu plus l'APRONUC : le retard dans les programmes de réhabilitation économique, une profusion d'initiatives diplomatiques des parrains du plan de paix, incohérentes pour ne pas dire concurrentes, et un comportement des personnels onusiens qui est loin d'être toujours moralement irréprochable. Face à cette réalité, n'oublions pas que les Khmers rouges disposent d'un appareil de propagande bien rôdé et savent apporter une réponse graduée aux menaces auxquelles ils doivent faire face. Tous les observateurs auront noté, par exemple, que chacune des mesures prises par les Nations-unies à leur encontre, pour ne pas avoir respecté scrupuleusement les accords de paix signés, se sont vues notifier un message militaire parfaitement mesuré. A l'heure où les Nationsunies votent les premières sanctions contre les Khmers rouges (résolution 783), deux ponts sautent sur la RN 21 et la RN 6. Le 7 novembre 1992, quelques heures avant l'ouverture de la réunion du Conseil National Suprême (CNS) à Beijing, c'est au tour d'un poste uruguayen d'être la cible des salves d'artillerie khmères rouges. Sans multiplier à l'infini les exemples, on soulignera également que le lendemain même de la décision d'embargo pétrolier (résolution 792), une unité de la PKD a capturé six officiers de l'APRONUC. Face à cette sophistication politico-militaire, qui entretien le mythe de la puissance khmère rouge, il conviendrait que le dispositif onusien soit parfaitement efficient, ce qui a l'évidence n'est pas le cas. Toutefois, cette violence symbolique n'est pas l'apanage seul des Khmers rouges. Bien que disposant de 50000 hommes ${ }^{6}$, l'armée de l'EdC limite son agressivité sur la vingtaine de divisions khmères rouges. Les quelques échanges d'artillerie et de mortier n'ont d'autres fonctions souvent que d'effrayer l'adversaire et l'ONU, pour empêcher notamment cette dernière 
d'accéder aux régions soumises à un strict contrôle politique. Il faut compter également sur l'auto-intoxication des acteurs pour expliquer certains accrochages. Ainsi les combats de la région de Phum Bavel peuvent s'interpréter par les craintes mutuelles des deux armées. Dans les rangs de l'EdC, on s'inquiète d'une offensive sur Battambang, qui paraît d'autant plus probable que la propagande officielle l'évoque à intervalle régulier depuis deux ans, tandis que les Khmers rouges sont convaincus que les effets d'annonce de l'EdC n'ont pour autre fonction que de cacher une attaque préemptive sur les bastions du mouvement avec la bienveillante neutralité des Nations-unies.

Face à ce bluff permanent, l'APRONUC a bien des difficultés à s'adapter aux nouvelles formes que prend le conflit. Au delà du sur-dimensionnement du dispositif militaire de l'APRONUC ( 3 militaires pour un civil), et compte tenu de la faible intensité des accrochages militaires, force est de constater les carences fonctionnelles du dispositif. A bien des égards, les unités militaires de l'APRONUC font preuve de légèreté et de faiblesse. Les militaires les plus expérimentés observent ainsi sur le terrain que les prises d'otages dans les régions de Kompong Thom et de Kratie ont laissé apparaître, à chaque fois, les erreurs commises par les soldats de l'APRONUC, peu habitués à adopter une posture dynamique, dans la planification des opérations de reconnaissance vers les zones khmers rouges et dans l'exécution tactique des missions. Malheureusement, pour accomplir leur mission de "peace keeping force", les Nations-unies disposent de bataillons dont chacun s'accorde à reconnaître un niveau tactique et technique insuffisant, voire un encadrement indigent. Pour éviter de perdre chaque jour un peu plus de sa capacité de dissuasion, il conviendrait que l'APRONUC sache faire preuve sur le terrain de détermination et de dynamisme. Trop souvent devant la menace d'affrontement militaire avec les unités de la PKD, les contingents de l'APRONUC battent en retraite. Pire même, des éléments indonésiens n'ont pas hésité à déposer leurs armes alors qu'ils avaient été mis en joue par des Khmers rouges. Comme le soulignait avec beaucoup d'humour un responsable de la composante militaire de l'APRONUC, à cette occasion ce n'était pas les Nations-unies qui désarmaient les hommes de Pol Pot mais les Khmers rouges qui désarmaient l'APRONUC. Cette preuve d'anémie n'est pas au demeurant très nouvelle et fut même avalisée de fait par les plus hautes instances de l'APRONUC, le 30 mai 1992, lorsque M.Akashi, accompagné du Lt Gal Sanderson, avait renoncé à pénétrer dans la zone de Païlin sur la simple injonction d'un garde barrière de la PKD. En d'autres termes, les Khmers rouges ont réussi à faire avaliser que le mandat onusien ne s'appliquait pas à l'ensemble du territoire cambodgien et dépendait de la bonne volonté des chefs de faction. Comme le souligne à juste titre C.Cheysson ${ }^{7}$, en adoptant une telle attitude, l'ONU vient de créer une nouvelle catégorie juridique de territoire en parlant de "zone inaccessible". Une telle appréciation revient à reconnaître que les Nations-unies ne pourront entrer sur les territoires khmers rouges que si elles en font la demande. Dans le cas contraire, l'APRONUC le ferait à ses propres risques et périls puisqu'elle "violerait" l'intégrité territoriale d'un des acteurs. Autant dire que les Nations-unies dépendent du bon vouloir des Khmers rouges! Une brèche dans laquelle la PKD s'est engouffrée très vite en déclarant, urbi et orbi, qu'elle peut intervenir sur tous les territoires du pays ${ }^{8}$.

Cette situation, de facto, est particulièrement inquiétante à l'heure où l'on découvre une directive khmère rouge, datant de mai 1992, visant à ce que les unités attaquent 30 à 40 villages par mois et exécutent sur le même laps de temps 10 à 20 Vietnamiens et/ ou officiels de l'EdC'. Sachant dorénavant que l'APRONUC peut être à son tour une cible potentielle, puisque les Khmers rouges la perçoive comme "une forme d'intervention 
dans la politique intérieure", la communauté internationale paraît d'autant plus faible que les Khmers rouges approchent de Phnom Penh des unités combattantes, pour faire preuve de leur puissance et accréditer l'idée que leur capacité militaire est encore efficace, voire intacte. Certes, certains évoquent les signes de démoralisation dans les rangs khmers rouges, notamment dans l'Est et le Nord-Est du pays, mais la défection des éléments les moins motivés est aussitôt compensée par l'arrivée de nouvelles recrues et n'est pas fondamentalement plus élevée que les années passées. Ce constat prouve si besoin était, que le mouvement dispose, au minimum, dans certaines franges de la société, d'une bienveillante neutralité des populations.

Mais si la PKD continue de gagner du temps pour développer ses zones d'influence et élargir sa base sociale, c'est à la fois grâce à la rémanence de son discours mobilisateur et à des moyens financiers considérables qui lui permettent de poursuivre la guerre, sans pour autant recourir à des pratiques strictement coercitives. En menant ce que J.F.Bayart qualifierait de "politique du ventre"10, les Khmers rouges engagent une véritable guerre économique qui pourra, peut être, servir un jour prochain le Parti de l'Union Nationale du Cambodge récemment formé par M.Khieu Samphan ${ }^{11}$. En effet, un des aspects les moins connu de la guerre au Cambodge, consiste à relever les déterminants économiques $d u$ conflit. Les affrontements récents ont notamment montré que toutes les factions cambodgiennes avaient intégré cette dimension dans leurs modes de guerre. Ainsi la combativité observée dans les affrontements de Choam Khsan se justifient, pour une bonne part, dans la volonté de conquérir ou de défendre un territoire connu pour ses riches potentialités. Plus inquiétant pour l'efficience des Nations-unies, il s'avère que dans certains endroits (ex. Ampil ${ }^{12}$ ou Sok San), les factions sont tellement militairement imbriqués que l'exploitation économique des territoires s'opère de façon concertée entre les mouvements armés. Si des intérêts économiques divergents suffisent parfois à entretenir la guerre, la volonté d'affamer les combattants adverses ou couper ses lignes de ravitaillement peuvent expliquer le plus souvent la rémanence des combats. Ainsi une des raisons du déploiement massif d'unités khmers rouges dans la région de Kompong Thom vise, depuis plusieurs mois, à couper les axes de ravitaillement gouvernementaux vers le Nord et le Nord Ouest du pays.

Le Khmer rouge : versus Homo economicus

7 Depuis l'annonce par Georges Bush, le 4 janvier 1992 à Singapour, de la levée effective de l'embargo économique américain, l'ensemble du territoire cambodgien est devenu un vaste champ de compétition. Tout s'achète, et un journaliste affirme même qu'on lui a proposé des missiles SAM-7 au tarif de $11000 \$$ pièce. Si cette brutale augmentation de la circulation monétaire provoque des envieux, force est de constater que le banditisme reste exceptionnel dans les zones khmères rouges, contrairement au reste du pays, où des unités de police en tenue, et avec brassards distinctifs, font régner l'ordre ${ }^{13}$. Cela ne signifie en rien que les 850 kilomètres de la frontière thaïlandaise, le long de laquelle le dispositif khmer rouge est adossé, soit exempte de trafics, bien au contraire, puisque toutes les factions profitent de l'anarchie dans l'Ouest et le Nord-Ouest du pays. Mais le dynamisme politico-militaire khmer rouge est particulièrement perceptible dans les différentes contrées bordant le Tonle Sap, régions entreprenantes et riches en ressources halieutiques (ex. les abords de Kompong Chhnang). Cette tactique permet au demeurant d'affaiblir le gouvernement de Phnom Penh en lui imposant la poursuite de la guerre et la gestion de milliers de personnes déplacées par les combats ${ }^{14}$, mais elle ne doit pas cacher que les recettes budgétaires du mouvement khmer rouge ont 
principalement pour origine le commerce des pierres précieuses (rubis, saphirs, émeraudes) et du bois extraits des territoires proches de la Thaïlande.

Bien qu'il soit très difficile de mesurer l'ampleur de l'enrichissement quotidien des Khmers rouges, une fourchette de un à quatre millions de dollars est parfaitement plausible ${ }^{15}$. En effet, pour l'heure, un tiers des rubis vendus sur le marché de Bangkok aurait pour origine le Cambodge. Ainsi, pas moins de 100000 personnes, dont un tiers de Birmaniens (parmi lesquels nombre de Môns), vivraient directement de cette activité économique des zones khmères rouges. Depuis les régions de Trat et Chanthaburi, six sociétés auraient ainsi investi près de dix milliards de baths pour extraire les pierres précieuses ${ }^{16}$. Outre la vente de droits de concession $(200000 \$$ plus $40000 \$$ par jour de prospection), les Khmers rouges bénéficieraient de $45 \%$ de la valeur des rubis extraits ${ }^{17}$. Ce sont les contrôleurs financiers khmers rouges qui fixent les prix, et en échange, ils garantissent la sécurité des opérateurs économiques dans leurs zones. Néanmoins, pour pouvoir accéder aux régions d'extraction et ramener la production en Thaïlande par les points frontaliers de Si Sa Ket, Surin, Prachin Buri, Trat, les sociétés exploitantes doivent reverser $5 \%$ du montant de leur production aux postes frontaliers. On peut incontestablement parler aujourd'hui d'une exploitation industrielle de ces mines d'autant plus intensive que la résolution 792 du 30 novembre 1992, dans son point 14, demande au CNS d'envisager de suspendre l'exploitation des minerais et des pierres précieuses $^{18}$. A la frontière, depuis le début de l'année 1991, la circulation monétaire est passée de 20 à 100 millions de baths chaque jour, au point que sur le marché de Ban Pakkard des négociants arriveraient à commercialiser plus de 100000 baths de pierres par jour. Cette exploitation systématique nécessite des matériels d'extractions modernes (200 gros tracteurs-extracteurs), de transports performants (240 camions à dix roues seraient en action) et des axes de communication entretenus ${ }^{19}$. Toutefois, il s'avère que ce négoce lucratif est relativement éclaté puisque 500 marchands thaïlandais commercent chaque jour avec les Khmers rouges ${ }^{20}$. Dans le même temps, les sommes engagées sont si importantes qu'elles nécessitent des protections politiques au plus haut niveau en Thaillande, au point que certains journalistes n'hésitent plus à écrire, à Bangkok, que le gouvernement démocratique, nouvellement élu, est sous le charme des hommes d'affaires ${ }^{21}$. Bien évidemment, il est très difficile de prouver de telles allégations mais on notera que parmi les hommes politiques qui défendent dans la presse le commerce frontalier, certains ont une bien mauvaise réputation. Ainsi un élu de Trat -Thanit Traiwut du Chart Pattana- serait un contrebandier notoire connu non seulement pour faire des affaires avec les Khmers rouges mais également avec le président de l'Assemblée nationale de l'EdC -Chea Simpour l'importation d'automobiles Toyota par le port de Koh Kong, une belle réussite qui pousse ses ramifications jusqu'aux marchés saïgonnais. Mais au delà de quelques politiciens véreux, ce sont les militaires qui bénéficient principalement de ces trafics puisque la frontière est sous leur entier contrôle et que s'y applique la loi martiale. Le plus inquiétant, comme le souligne l'éditorialiste Kavi Chongkittavorn, c'est que "les décideurs politiques au plus haut niveau misent sur une victoire des Khmers rouges dans l'avenir, soit par les moyens politiques, soit par les armes. Les militaires ont peur des représailles des Khmers rouges, et c'est pour cette raison qu'ils préfèrent le statu quo !" 22.

9 La coupe de bois est la deuxième source significative de revenu des Khmers rouges, rapportant, in fine, 5 à 8 dollars le m3. Néanmoins comme le souligne une étude du PNUD, révélée lors du Sommet de la Terre à Rio, toutes les factions khmères peuvent 
être accusées de dilapider la richesse nationale ${ }^{23}$. En 1992, pas moins de 1,15 millions de m3 de bois ont été ainsi coupés dont $320000 \mathrm{~m} 3$ ont été exportés par l'EdC, 200000 par la PKD, 128000 par le FUNCINPEC et 50000 par le FNLPK. Les Khmers, bradant souvent le prix du bois, les sociétés thaïlandaises sont les premières à se porter acquéreur de ce produit. Les 26 sociétés thaïs opérant sur le terrain sont d'autant plus motivées à le faire que depuis janvier 1989, le Premier ministre thaïlandais a banni l'exploitation forestière dans son pays. Le moment est venu de "mettre en valeur" le Cambodge comme ce fut le cas avant lui du Myanmar ${ }^{24}$ et du Laos. Toutefois, si nous percevons mieux actuellement l'ampleur du phénomène ${ }^{25}$, il n'est pas pour autant récent Dès juin 1989, le commandant de la 302ème division d'infanterie khmère rouge signait un contrat d'exploitation avec la société thaïe, Ruamphana, connue pour disposer du soutien de députés du Chart Thai et plus largement du Département Royal des Forêts.

Compte tenu du caractère particulièrement lucratif de ce marché, on comprend que les exploitants aient tenté de persuader l'APRONUC d'adopter un moratoire de six mois pour renoncer à l'interdiction d'exportation prise par le CNS, le 22 septembre 1992. Malgré l'entrée en vigueur de cette restriction le 1er janvier 1993, l'embargo est ouvertement violé pour pouvoir rentabiliser des investissements moyens de 10 à 20 millions de baths, plus une concession acheté 60 à 70 millions. Les militaires sont ici aussi les premiers bénéficiaires de cette activité économique dont la moitié de la production a été exportée en Thaïlande ${ }^{26}$. La rumeur publique évoque les noms de plusieurs généraux (ex.Gal Issarapong) et personnalités du ministère de l'Intérieur parmi les opérateurs économiques tandis que les unités frontalières perçoivent leur dîme à l'image de l'Unité 838, responsable du renseignement, dont les éléments préleveraient une taxe de 50 baths pour chaque mètre cube importé.

11 En "bon" gestionnaire, les Khmers rouges ont anticipé les perturbations du marché qui pouvaient naître de la mise en oeuvre de l'embargo commercial décidé par le CNS. Certes, le 14 janvier 1992, le gouvernement de Bangkok a déjà interdit les importations de bois du Cambodge faites sans licence mais cette décision est restée sans effet, si ce n'est de permettre aux Khmers rouges d'infiltrer encore plus dans les réseaux économiques thaïs. Toutefois, pour faire face aux menaces présentes des Nations-unies, les Khmers rouges mènent une véritable politique des prix, visant à abaisser le loyer des concessions, voire en certains lieux à exempter les compagnies minières de verser des redevances aux villageois du lieu d'exploitation, afin d'encourager les sociétés à rester. A contrario, les Khmers rouges demanderaient aux personnels d'amener avec eux du riz et de la nourriture pour faire face aux sanctions, complétant ainsi les stocks de nourriture constitués dans cette perspective. A plus long terme, il s'avérera très difficile de mettre un terme à ces pratiques d'exploitations illicites. Même dans les sociétés démocratiques d'Asie du Sud-Est, l'arrêt de l'exploitation forestière illégale n'est pas des plus aisées. Aux Philippines, seulement, 0,5\% de la population considère qu'il s'agit ainsi d'améliorer la justice dans le pays ${ }^{27}$. De surcroît, outre les pertes fiscales qu'engendrent pour le gouvernement de telles pratiques, on relève déjà des dégâts écologiques perceptibles sur le bassin fluvial de Battambang et plusieurs rivières frontalières. Plus inquiétant à long terme, ce mode de développement économique ne fait qu'aggraver le déboisement d'un pays qui a vu sa couverture forestière passer de 73\% du territoire en 1965 à 40\% en 1992.

12 L'ensemble de ces pratiques économiques relativement sophistiquées ne doivent pas cacher la poursuite de certaines formes d'actions violentes notamment lorsque les 
Khmers rouges réquisitionnent du riz et des charrettes pour le transporter. Néanmoins, les sommes dégagées par les pratiques commerciales les plus modernes permettent au mouvement d'avoir une certaine autonomie et de subvenir pour l'essentiel à ses besoins quotidiens. Contrairement aux pratiques de prévarication de Phnom Penh, les liquidités khmères rouges servent à alimenter l'action sociale de la guérilla (achats de médicaments, dons aux pagodes, aides de proximité...) susceptibles d'entretenir l'image d'un mouvement insurrectionnel proche des pauvres. Il est à craindre que, dans la perspective électorale, cet argent soit également utilisé à des fins de (re)construction de réseaux d'allégeances politiques (achats de voix et de candidats) sur l'ensemble du territoire. Même si nous ne disposons que d'une vision très approximative des moyens effectivement dépensés, voire des canaux utilisés, une telle hypothèse paraît parfaitement s'inscrire dans la stratégie de reconquête du pouvoir à long terme du mouvement khmer rouge. Il n'en demeure pas moins que la redistribution fiduciaire ne se pratique pas uniformément dans toutes les unités territoriales khmères rouges. Les structures politico-militaires, dispersées sur le territoire, vivent souvent en autarcie économique sans disposer semble-t-il d'une aide quelconque des organes centraux du mouvement. Mais quel que soit le niveau de pauvreté de ces unités, et malgré l'existence d'un début de dialogue avec les éléments de l'APRONUC, force est de constater la permanence d'une très grande détermination et d'une grande fidélité politique des combattants et de leurs familles.

Dans le même temps, il apparait qu'une partie des sommes, qui ne sont pas dépensées, sont épargnées à l'étranger. Des banques thaïlandaises, chinoises, en Asie du Sud-Est où ailleurs, sont le plus souvent utilisées. Une partie de cet argent est investi en Occident voire à l'intérieur de l'économie de l'EdC. A Phnom Penh, des clabauderies évoquent, ici où là, des investissements suspects dans le secteur commercial, le tourisme ou encore les transports internationaux. Alors que certains investissements asiatiques au Cambodge peuvent servir à blanchir de l'argent né de pratiques mafieuses, ils peuvent tout autant servir de paravent à une certaine forme de transferts financiers pour le compte des Khmers rouges.

Même s'il n'est plus rare de croiser dans les zones "libérées" des cadres khmers rouges en Mercedes, on ne peut pas affirmer encore que nous assistions à une corruption politique du mouvement. Néanmoins, Ta Mok, l'un des leaders les plus importants, possèderait quant à lui, dans le district de Ku Khan (Thaïlande), une vingtaine $d$ 'hectares de terrains, plusieurs stations services d'essence et un réseau de distribution de machines agricoles. Il est encore trop tôt cependant pour valider la conviction de ceux qui pensent qu'un dialogue serait possible avec les éléments les plus ouverts de la direction de la PKD, qui "croiraient aux vertus de commerce plus qu'à celles de la guerre". Certes, on remarquera, par exemple, que le commandant de la 519ème division khmère rouge est devenu lui aussi un homme d'affaires indépendant mais rien ne prouve que la corruption politique du mouvement dans son ensemble soit très avancée ou en voie de généralisation. De la même manière, l'interception récente de quelques kilogrammes de marijuana ne permet pas d'affirmer que nous assistons peu à peu à une criminalisation du mouvement khmer rouge comme ce fut le cas d'autres guérillas en Asie du Sud-Est (Birmanie, Malaysia, Sumatra). Ceteris paribus, ces dérives économiques demeurent, somme toute, modestes au regard de la concussion et de la corruption institutionnalisée de l'EdC. 
15 Même si la radio khmère rouge relaie les propos du Prince Norodom Sihanouk affirmant que l'économie est dorénavant sous le contrôle de l'étranger et des grandes puissances, expliquant ainsi l'exploitation intensive et extensive des ressources naturelles ${ }^{28}$, c'est la Thaïlande qui est aujourd'hui la nation prédatrice. Les Khmers rouges, au nationalisme sourcilleux, sont ainsi à leur tour des instruments de la présence étrangère au Cambodge. M.Khieu Samphan en dénonçant, la monnaie nationale comme un instrument permettant la poursuite de l'occupation vietnamienne fragilise un peu plus l'économie locale et favorise la substitution du riel par le dollar américain, l'or et le bath thaïlandais. Il semble en effet que ces trois monnaies soient les instruments de négoce des Khmers rouges. Même s'il paraît pour le moins osé de croire que les Khmers rouges soient les seuls instruments de la déstabilisation monétaire, les rumeurs sur l'introduction de faux billets de 500 riels par les Khmers rouges dans les circuits financiers accélèrent les phénomènes d'éviction. Une menace prise très au sérieux par l'EdC pour lequel " le refus d'utiliser les billets de banques est un acte de sabotage "29. Pour l'heure, tout est important en matière économique pour les Khmers rouges. C'est pourquoi ils s'opposent tour à tour à une intervention du $\mathrm{FMI}^{30} \mathrm{ou}$ réclament que l'or et l'argent en dépôt au Cambodge soit placé sous le contrôle du CNS, plutôt que de servir à payer l'endettement de l'EdC et s'avèrent menaçant en évoquant une reprise de la guerre générale ou le développement du banditisme dans les provinces thaïlandaise de Buri Ram et Prachin Buri ${ }^{31}$. Dans un tel contexte, comment s'étonner dès lors que les commentateurs thaïlandais fassent valoir que l'obstacle principal à la paix au Cambodge est la présence vietnamienne. Sinchai Vorasart soupçonne même le Viet Nam d'être derrière l'interdiction de l'exploitation du bois cambodgien faite aux commerçants thaïlandais ${ }^{32}$. Le vice-président de la commission des affaires étrangères de l'Assemblée nationale -Prapas Lipabhamdu ${ }^{33}$ - va plus loin quant à lui en accusant ouvertement l'APRONUC d'avoir manqué à sa neutralité depuis sa mise en place, et ajoute que dans ces conditions la réaction des Khmers rouges lui paraît d'autant plus légitime que l'appareil de l'Etat du Cambodge, investi et dominé par la faction Hun Sen, s'est employé à tenir écarté les autres factions de l'administration provisoire du pays. Si à Bangkok, nombreux sont ceux qui comme le général Vimol sont convaincus que l'ONU cherche à pallier son échec en impliquant la Thaïlande dans leur querelle avec la $\mathrm{PKD}^{34}$, elle peut se satisfaire de la présente situation car elle exerce un véritable monopole économique que ne lui permettrait pas une stabilité politique du Cambodge et son ouverture au monde. Dans ce contexte, il convient de s'interroger sur le point de savoir si des sanctions efficaces peuvent être prises contre les khmers rouges, si elles doivent être morales, symboliques ou réellement contraignantes. Avant d'en prendre, il faut donc s'assurer de leur efficacité. C'est pourquoi, il est à craindre que la résolution 766 du 21 juillet 1992 qui prévoit la réservation de l'aide internationale aux parties coopérant avec l'ONU ne pourra suffire. A l'heure où en Thaïlande quelques individualités demandent au Ministère des affaires étrangères d'expliquer de façon plus claire à la communauté internationale ses rapports avec les Khmers rouges et de lever tout soupçon de la part des autres factions khmères ${ }^{35}$, il conviendrait d'éviter que les Khmers rouges ne mettent à profit les insuffisances de l'Etat du Cambodge et de l'APRONUC pour augmenter, petit à petit, leur présence. C'est en faisant face aux problèmes économiques de la nation cambodgienne (il s'est avéré nécessaire d'importer 200000 tonnes de riz en $1992^{36}$ ) et en mettant au plus vite en action les programmes de réhabilitation économique programmé lors de la Conférence de Tokyo que la communauté internationale pourra 
assurer un minimum de stabilité au pays tout en évitant sa partition entre deux voisins compradores.

\section{NOTES}

1. Cette étude s'inscrit dans le cadre d'une recherche menée avec l'association Les amis d'une Ecole de la Paix (Grenoble).

2. Kershaw (I.) : Qu'est-ce que le nazisme ? : Problèmes et perspectives d'interprétation, Gallimard, 1992, 416 p ; Marc Nouschi : Histoire des salauds, Editions n ${ }^{\circ} 1,1992,214$ p ;

Guy Richard : L'histoire inhumaine : massacres et génocides des origines à nos jours, Armand Colin, 1992, $480 \mathrm{p}$

3. Sliwinski (M.) : Evaluation des conséquences démographiques et sociales de la guerre et la révolution des Khmers rouges au Cambodge ( 1970 / 1989 ), Genève, novembre 1992, $140 \mathrm{p}$ (multigraphié).

4. Kyodo 2 décembre 1992

5. Lechervy (C.), "Cambodia : A New Reading of the Conflict", Colloque IFRI - JIIA, Tokyo, 11/12 janvier 1993 ( à paraître )

6. L'armée de l'EdC compte 112000 hommes y compris les forces provinciales et de districts.

7. Propos tenu par l'ancien ministre français des relations extérieures, après une mission du Parlement européen, lors du colloque L'Etat du Cambodge en 1993, France Asia Press, Arche de la Fraternité, 19 décembre 1992.

8. Si l'on en croit la propagande khmère rouge il faut distinguer trois types de territoires militaires au Cambodge. Dans les régions libérées et organisées (zone 1) ou semi-libérées (zone 2), les Khmers rouges peuvent assurer à 100\% la protection de leurs "invités", sous entendus les Nations-unies-. A contrario, en zone 3, région d'activité des guérillas, les Khmers rouges ne pourraient garantir cette sécurité qu'à $50 \%$. Voice of the Great National Union Front of Cambodia 22 décembre 1992 -- SWB FE/1572 B/1 9. Kyodo 14 décembre 1992

10. Bayart (J.F.), L'Etat en Afrique : la politique du ventre, Fayard, 1989, $439 \mathrm{p}$

11. Le PNUCD installé à Païlin comprendrait par ordre d'importance outre Khieu Samphan, MM Son Sen, Chan Youran, Sar Kim Lmut, Hing Un, Pech Bunret, In Sopheap, Mak Ben, Kor Bun Heng et Tep Khunnal. Voice of the Great National Union Front of Cambodia 29 novembre 1992 -- SWB FE/1552 B/2 - B/3

12. Ancienne capitale des territoires libérés sihanoukistes.

13. Selon les Khmers rouges, le banditisme au Cambodge est surtout dû à l'absence de soutien populaire au régime de Phnom Penh et plus généralement le fait des Vietnamiens. Voice of the Great National Union Front of Cambodia 19 octobre 1991 -SWB FE/1519 B/3

14. A l'automne 1992, les combats dans la région de Phun Bavel ont provoqué la fuite de 12000 personnes vers les territoires de l'EdC. Il est évident que toute aide de la communauté internationale à ces populations sera dénoncée comme une preuve de la partialité de l'APRONUC apportant un soutien implicite à l'EdC. 
15. Finantial Times 12 novembre 1992, Le Point 21 novembre 1992, Libération 1er décembre 1992

16. $1 \mathrm{FF}=4,8$ Baths

17. Ces valeurs numériques permettent de penser que le revenu mensuel des Khmers rouges grâce aux autorisations de prospection serait de 2 millions de dollars par mois. Nous sommes donc bien des chiffres de revenus annoncés par les officiels thaillandais. En juin 1992, celles-ci affirmaient que le revenu mensuel total se situait à un million de dollar par mois.

18. Dans un tel cas de figure, toutes les factions risquent d'être pénalisées. La rumeur publique veut que des proches de l'entourage de M.Hun Sen soient impliqués dans des trafics de diamant et d'or tandis que dans la province de Mondolkiri, les mines d'or et de pierres précieuses attirent les migrants vietnamiens.

19. Un journaliste relevait ainsi que pour la saison des pluies, on avait entretenu les routes avec des conduits d'évacuation Agence France Presse 30 novembre 1992

20. Bangkok Post 17 novembre 1992

21. The Nation 14 novembre 1992

22. Agence Cambodge Laos $n^{\circ} 27$, octobre-novembre 1992 -- The Nation 13 novembre 1992

23. Selon certains responsables du FUNCINPEC, les contrats forestiers auraient été suspendus à la fin 1992.

24. Dilapidation du patrimoine forestier in C.Lechervy : Birmanie à un tournant, PPS n692, 27 novembre 1992

25. Les coupes de bois se faisant le plus souvent d'octobre à avril, cet intervalle de temps intervient au moment du déploiement optimum des unités civiles et militaires de l'APRONUC.

26. Même si depuis 1987, des Thaïlandais font également du commerce de bois sur la frontière vietnamienne ( Bangkok Post 15 novembre 1992 ). Ainsi, la moitié du bois exporté par le Viet Nam, en 1991, aurait pour origine le Cambodge et serait acheminé principalement par la route qui relie la province de Ratanakiri à celle de Gia LaiKantum. On retiendra également qu'une partie du bois importé officiellement du Cambodge est parfois tout simplement coupé illégalement en Thailande.

27. Social Weather Stations Survey, Université de l'Aténéo, Manille, septembre 1992. The Manila Chronicle 16 novembre 1992

28. Voice of the Great National Union Front of Cambodia 14 décembre 1992. SWB FE/ $1565 \mathrm{~B} / 2$

29. Pracheachon 26 juillet 1992

30. Voice of the Great National Union Front of Cambodia 30 octobre 1992. SWB FE/1529

$\mathrm{A} 1 / 4$

31. Bangkok Post 23 août 1992

32. Naewna 14 décembre 1992

33. Ancien vice-ministre des affaires étrangères.

34. The Nation 22 décembre 1992

35. Matichon 15 décembre 1992

36. Pour mémoire, en 1937, le Cambodge exportait 400000 tonnes de riz. 


\section{RÉSUMÉS}

Même si les Khmers rouges ne semblent pas être en mesure de prendre, ou plutôt de reprendre actuellement le pouvoir au Cambodge, on ne saurait cependant les sous-estimer, se serait une faute politique grave selon Christian Lechervy, tant la sophistication de leur organisation politique, la cohérence de leur discours de propagande et leurs capacités à s'adapter aux nouveaux contextes post-bipolaires sont grandes. Il faut toutefois se garder de voir uniquement dans les Khmers rouges un monstre sanguinaire. La faiblesse, voire l'incohérence des forces militaires onusiennes (APRONUC), véritablement "bluffées" par les Khmers, mais également l'incapacité du gouvernement de Phnom Penh à "réagir", ne sont pas étrangères, loin s'en faut, à la "renaissance" du mouvement. Si l'aspect militaire du conflit ne peut être nié, l'auteur met particulièrement l'accent sur ses déterminants économiques, le Cambodge devenant ainsi pour tous les acteurs un vaste champ de compétition économique.

Even though the Khmer Rouges do not seem presently capable of assuming political power in Cambodia, their potential influence should not be underestimated : their political organisation is sophisticated, their propaganda coherent and they are capable of adapting themselves to the new post-bipolar situation. It would be wrong to consider the Khmer Rouges exclusively as bloodthirsty monsters. The revival of the movement is due, to a large extent, to the weakness, or perhaps incoherence, of the UN forces, which have been " fooled " by the Khmer Rouges, and to the incapacity of the Phnom Penh government to react. Without forgetting the military aspect of the conflict, the author centres on the economic factors which have turned Cambodia into a game-field of opposing economic interests.

\section{INDEX}

Index géographique : Asie du Sud-Est, Cambodge

Mots-clés : économie politique, guérillas et organisations clandestines, maintien de la paix, sortie de conflits

Thèmes : Khmers rouges 